\title{
Supplimentary: Strong Magnetic Response of Optical Nanofibers
}

\author{
Shaghik Atakaramians, ${ }^{*} \dagger$ Andrey E. Miroshnichenko, ${ }^{\ddagger}$ Ilya V. Shadrivov, ${ }^{\ddagger}$ Ali \\ Mirzaei, ${ }^{\ddagger}$ Tanya M. Monro, $₫$ Yuri S. Kivshar, $\ddagger$ and Shahraam Afshar V. \\ $\dagger$ Institute of Photonics and Optical Science, School of Physics, The University of Sydney, \\ Sydney NSW 2006, Australia \\ $\ddagger$ Nonlinear Physics Centre, Research School of Physics and Engineering, Australian \\ National University, Canberra ACT 2601, Australia \\ ILaser Physics and Photonic Devices Laboratories, School of Engineering, University of \\ South Australia, Mawson Lakes SA 5095, Australia \\ Institute for Photonics and Advanced Sensing, The University of Adelaide, Adelaide SA \\ 5005, Australia. \\ E-mail: shaghik.a@sydney.edu.au
}

\section{Multipole expansion}

Electromagnetic multipole expansion is used to decompose the electric field of the system on a sphere in terms of localized sources, i.e. electric charges and currents. ${ }^{1,2}$ The system is consists of an electric dipole excited at $\lambda=700 \mathrm{~nm}$ placed at the core-clad interface (on the $x$-axis) of an air-clad nanofiber, with telluride $(n=2.025)$ as the hosting material. The fiber is oriented along the $z$-axis and the center of the coordinates is at the center of the fiber cross section as shown in the insets of Figure 1. The electric field of the system can be written in spherical coordinates in the form of mulitpole expansion using eq. 2. When the electric 
field is known on a sphere (e.g. calculated numerically), the multipole coefficients $a_{E}(l, m)$ and $a_{M}(l, m)$ can be derived using the orthogonality properties of the spherical harmonics as follows: ${ }^{2}$

$$
\begin{gathered}
a_{E}(l, m)=\frac{(-i)^{l+1} k r}{h_{l}^{(1)}(k r) E_{0} \sqrt{\pi(2 l+1) l(l+1)}} \int_{0}^{2 \pi} \int_{0}^{\pi} Y_{l m}^{*}(\theta, \phi) \hat{\mathbf{r}} \cdot \mathbf{E}_{\mathbf{s}}(\mathbf{r}) \sin \theta d \theta d \phi \\
a_{M}(l, m)=\frac{(-i)^{l}}{h_{l}^{(1)}(k r) E_{0} \sqrt{\pi(2 l+1)}} \int_{0}^{2 \pi} \int_{0}^{\pi} \mathbf{X}_{l m}^{*}(\theta, \phi) \cdot \mathbf{E}_{\mathbf{s}}(\mathbf{r}) \sin \theta d \theta d \phi
\end{gathered}
$$

where $\mathbf{X}_{l m}$ and $Y_{l m}$ are the normalized vector and scalar spherical harmonics respectively, $h_{l}^{(1)}$ is the spherical Hankel functions of the first kind, and $\mathbf{E}_{s}$ is the scattered electric field. Figure S1 shows the coefficients $a_{E}(l, m)$ and $a_{M}(l, m)$ at the position of the first peak for (a) $z$ - and (b) $r$-oriented excitations. Only positieqnarrayve $m$ multipole components are shown, due to the symmetry of $a_{E, M}(l, m)$ and $a_{E, M}(l,-m)$ components. It is evident that the electric multipoles are enhanced for the system, $a_{E}(1,0)$ and $a_{E}(1,1)$ for respectively $z$ and $r$-oriented excitations, in comparison with the source only.

The Cartesian multipole moments for the system can be calculated from the coefficients $a_{E}(l, m)$ and $a_{M}(l, m) .{ }^{2}$ The Cartesian dipole moments of the system in terms of $a_{E}(1, m)$ and $a_{M}(1, m)$ shperical coefficients are as follows: ${ }^{2}$

$$
\begin{aligned}
& \left(\begin{array}{c}
p_{x} \\
p_{y} \\
p_{z}
\end{array}\right)=C_{p}\left(\begin{array}{c}
{\left[a_{E}(1,1)-a_{E}(1,-1)\right]} \\
i\left[a_{E}(1,1)+a_{E}(1,-1)\right] \\
-\sqrt{2} a_{E}(1,0)
\end{array}\right) \\
& \left(\begin{array}{c}
m_{x} \\
m_{y} \\
m_{z}
\end{array}\right)=C_{m}\left(\begin{array}{c}
{\left[a_{M}(1,1)-a_{M}(1,-1)\right]} \\
i\left[a_{M}(1,1)+a_{M}(1,-1)\right] \\
-\sqrt{2} a_{M}(1,0)
\end{array}\right)
\end{aligned}
$$

where $C_{p}=-i\left(3 \pi \epsilon E_{0}\right) / k^{3}, C_{m}=-i\left(6 \pi \epsilon E_{0}\right) / k^{4}$, and $\left(p_{x}, p_{y}, p_{z}\right)$ and $\left(m_{x}, m_{y}, m_{z}\right)$ are respectively the Cartesian electric and magnetic dipole moments. 

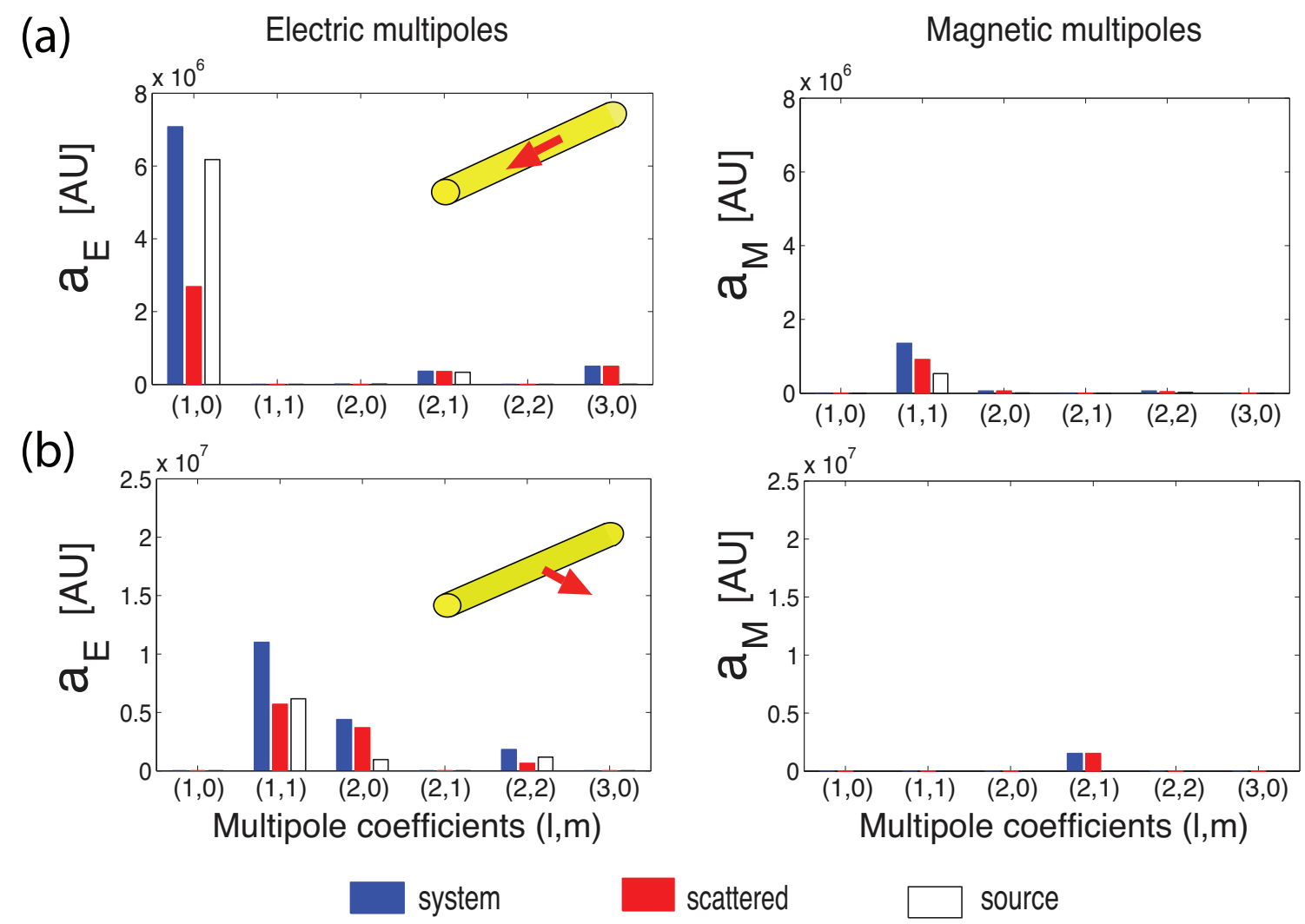

Figure S1: The electric and magnetic multipole coefficients of total electric field of the system at the position of the first radiation peak for (a) $z$ - and (b) $r$-oriented excitations.

Figure S2a shows the Cartesian ED and MD moments of a $\phi$-oriented electric dipole located on the $x$-axis (off centered $x$-oriented). The results confirm a strong ED moment along $y$ direction. By presence of the fiber, the ED component at the position of the first peak is suppressed dramatically, while the $\mathrm{MD}$ component is enhanced by $\approx 1.8$. Figure $\mathrm{S} 2 \mathrm{~b}$ shows the relative contribution of electric $\left(C_{E}(l, m)\right)$ and magnetic $\left(C_{M}(l, m)\right)$ mulitpoles in the total energy of the system. Due to symmetry of $C_{E, M}(l, m)$ and $C_{E, M}(l,-m)$ components, the total contribution of both components is presented at $(l, m)$. It shows that the total enegry of the system is mainly in $(1,0)$ magnetic component, while for the source only it is mainly in $(1,1)$ electric component. This demonstrates that it is possible to suppress the electric response and preferentially enhance the magnetic response of coupled dipole-fiber source so that $C_{M}(1,0) / 2 C_{E}(1,1) \sim 112$ for the system. This strong magnetic response is observed in a system with relatively low refractive index compared to that reported earlier 
for high-index dielectric nanoparticles.


(b)

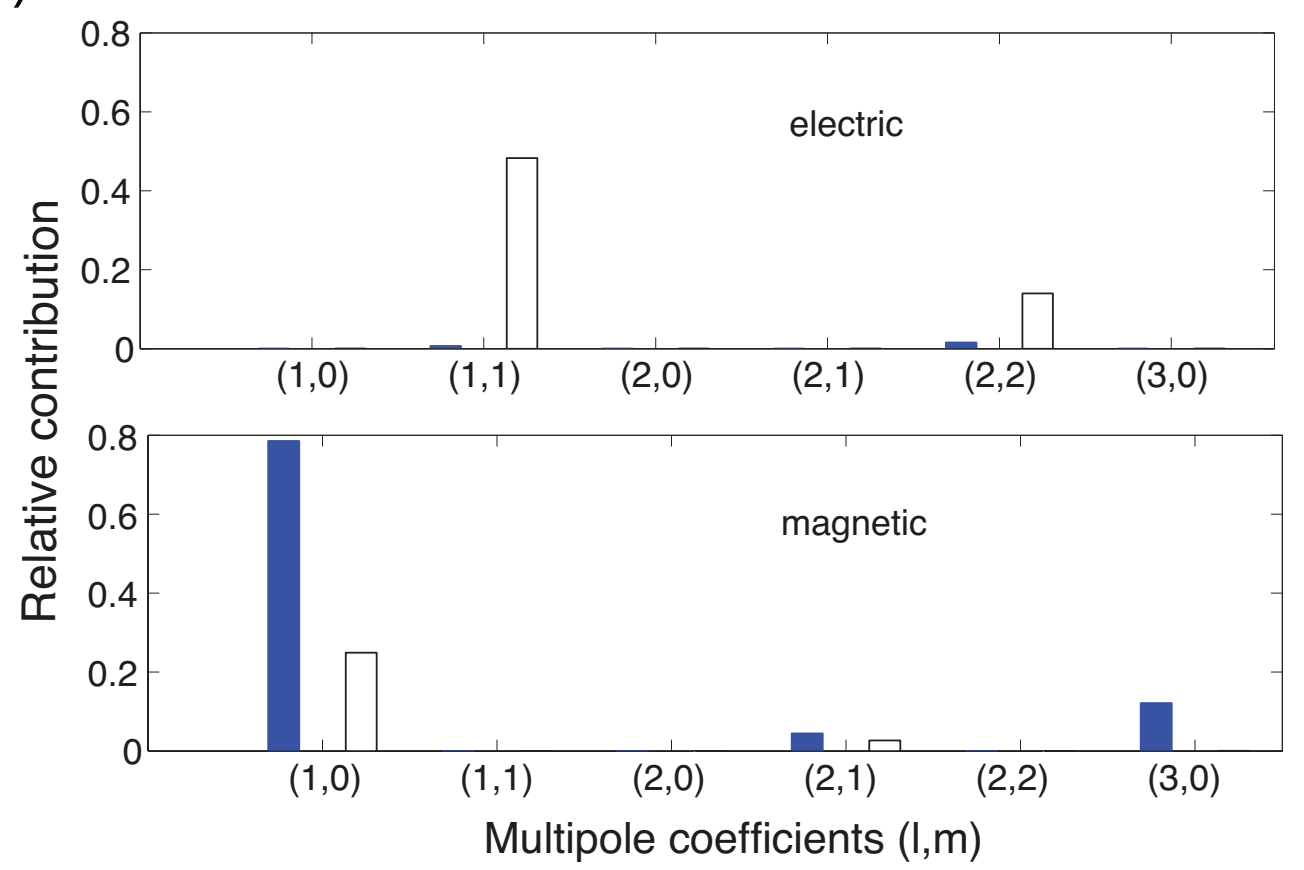

Figure S2: (a) Cartesian ED and MD moments and (b) relative contribution of elctric and magnetic multipoles in the total enegry of the system at the postion of the first radiation peak for a $\phi$-oriented dipole excitation. 


\section{Numerical modelling}

We use CST microwave studio to calculate the far field pattern of the coupled dipole-fiber system at the position of the first peak. Figure S3a shows the far field power pattern for a $z$-oriented excitation. The fiber breaks the symmetry and adds a weak directionality to the radiated power. For a $\phi$-oriented excitation (Figure S3b), the pattern is formed by an interference of a strong magnetic dipole and a magnetic quadrupole. This results into appearance of additional zeros at $z$ direction, suppression of power in $x$ direction and redirection of the power into narrow beams in the plane perpendicular to the source.
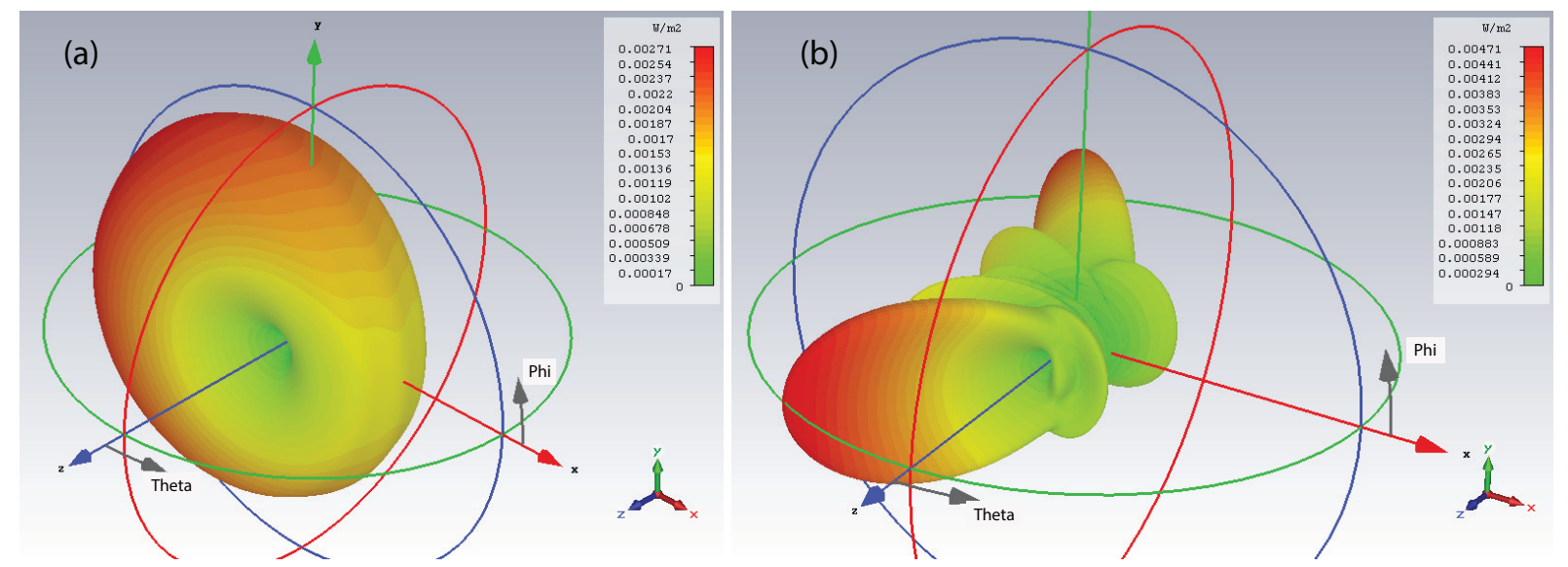

Figure S3: The far field power pattern of the system at the position of the first radiation peak for (a) z-and (b) $\phi$-oriented excitations.

\section{References}

(1) Jackson, J. D. Classical Electrodynamics, 3rd ed.; John Wiley \& Sons, Inc., 1998.

(2) Grahn, P.; Shevchenko, A.; Kaivola, M. Electromagnetic multipole theory for optical nanomaterials. New J. Phys. 2012, 14, 093033. 